\title{
Beam divergence measurements of InGaN/GaN micro-array light-emitting diodes using confocal microscopy
}

\author{
C. Griffin, ${ }^{\text {a) }}$ E. Gu, H. W. Choi, C. W. Jeon, J. M. Girkin, and M. D. Dawson \\ Institute of Photonics, University of Strathclyde, 106 Rottenrow, Glasgow G4 ONW, United Kingdom \\ G. McConnell \\ Centre for Biophotonics, University of Strathclyde, 27 Taylor Street, Glasgow G4 ONR, United Kingdom
}

(Received 24 February 2004; accepted 12 November 2004; published online 20 January 2005)

\begin{abstract}
The recent development of high-density, two-dimensional arrays of micrometer-sized InGaN/GaN light-emitting diodes (micro-LEDs) with potential applications from scientific instrumentation to microdisplays has created an urgent need for controlled manipulation of the light output from these devices. With directed light output these devices can be used in situations where collimated beams or light focused onto several thousand matrix points is desired. In order to do this effectively, the emission characteristics of the devices must be fully understood and characterized. Here we utilize confocal microscopy to directly determine the emission characteristics and angular beam divergences from the individual micro-LED elements. The technique is applied to both top (into air) and bottom (through substrate) emission in arrays of green $(540 \mathrm{~nm})$, blue $(470 \mathrm{~nm})$, and UV $(370 \mathrm{~nm})$ micro-LED devices, at distances of up to $50 \mu \mathrm{m}$ from the emission plane. The results are consistent with simple optical modeling of the expected beam profiles. (C) 2005 American Institute of Physics. [DOI: 10.1063/1.1850599]
\end{abstract}

Several groups have reported recently on the development of high-density, two-dimensional arrays of micrometersized InGaN/GaN light-emitting diodes. ${ }^{1-3}$ The latest advances $^{3}$ have provided matrix-addressable, highperformance arrays of up to $\sim 12000,20$ - $\mu \mathrm{m}$-sized emitters at $1200 \mathrm{dots} / \mathrm{in}$. The ability to program the emission patterns of these arrays, together with the important spectral coverage they offer across the $370-600 \mathrm{~nm}$ range (and potentially 280-370 nm with Al-containing III-N alloys), opens up a wide range of scientific and instrumentation uses for these devices. Examples include novel microdisplays ${ }^{1}$ and excitation sources for micro-array format fluorescently tagged biomolecules. ${ }^{4}$ Biological and chemical applications of these light-emitting diode (LED) arrays are of particular interest as they provide an opportunity to develop multiwell plate readers in fluorescence lifetime assays.

To form a useful illumination source for these purposes it is necessary for the light from each element to be precisely imaged, collimated, or manipulated for projection onto an applications plane. Arrays of refractive microlenses positioned in registry with each emitter element can in principle offer this control, and preliminary microlens formation with selected compatible materials (polymers, ${ }^{2} \mathrm{GaN},{ }^{5}$ sapphire ${ }^{6}$ ) have been reported for such a purpose. To accurately collect and manipulate the emission in this manner, however, requires a full knowledge of the light emission characteristics of the micro-LED elements. Here, we report a detailed study of these characteristics for blue, green, and ultraviolet microLED arrays, respectively. We have utilized confocal microscopy for these measurements, and determined that the predominant emission pattern both upwards (into air) and downwards (toward the substrate) is confined to a cone whose characteristics are accurately determined by this

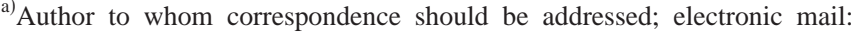
chris.griffin@strath.ac.uk
}

method. Confocal microscopy has been used previously to characterize the photoluminescence of GaN-related devices. ${ }^{7}$

The micro-LEDs studied were $64 \times 64$ arrays of microdisk elements, each $20 \mu \mathrm{m}$ in diameter, within an overall active footprint of $3 \mathrm{~mm} \times 3 \mathrm{~mm}$, and were matrixaddressable via a contact grid. The wafer details and fabrication process for the blue and green devices has been described previously. ${ }^{3}$ The key processing step is the production of sloping sidewalls in the InGaN/GaN micropillars by inductively coupled plasma dry etching, to facilitate conformal metal coverage. The resulting truncated conical emission elements were sheathed by metal on their sidewalls. The ultraviolet devices ${ }^{8}$ were fabricated by the same basic approach, and their electrical and power performance details will be reported in full elsewhere. All structures were grown on 2 in. $c$-plane sapphire substrates by metalorganic chemical vapor deposition. The ultraviolet structure, not described previously in this context, consists of $2.3 \mu \mathrm{m}$ of undoped $\mathrm{GaN}$ and $1.4 \mu \mathrm{m}$ of $n$-doped GaN, over which was a $200 \mathrm{~nm}$ cladding layer and a seven-period multiquantum well, capped with $100 \mathrm{~nm}$ of $\mathrm{Mg}$-doped AlGaN cladding and a $20 \mathrm{~nm} \mathrm{Mg}$-doped $\mathrm{GaN}$ contact layer. The quantum well was of seven periods, of $2 \mathrm{~nm} \mathrm{InGaN} \mathrm{(In}$ $\sim 0.05$ ) and $10 \mathrm{~nm} \mathrm{AlGaN} \mathrm{(Al \sim 0.2)} \mathrm{barriers.} \mathrm{The} \mathrm{blue,}$ green, and ultraviolet arrays were operated with $3.4,4$, and $12 \mathrm{~V}$ with respect to forward bias voltages at around $1 \mu \mathrm{A}$. These values were chosen to be above the turn on voltages, to produce sufficient detectable light output from the devices. The blue device was also studied at $4 \mathrm{~V}$ to determine whether a higher voltage altered the beam divergence.

The emission characteristics of individual micro-LED elements were determined using a commercial confocal microscope, as illustrated in Fig. 1. A commercial confocal scan head was connected to an upright microscope equipped with a $\times 20,0.75$ n.a. objective lens. The emission from the electrically excited LED elements was imaged back through the confocal scan head and detected on one of the three photo- 


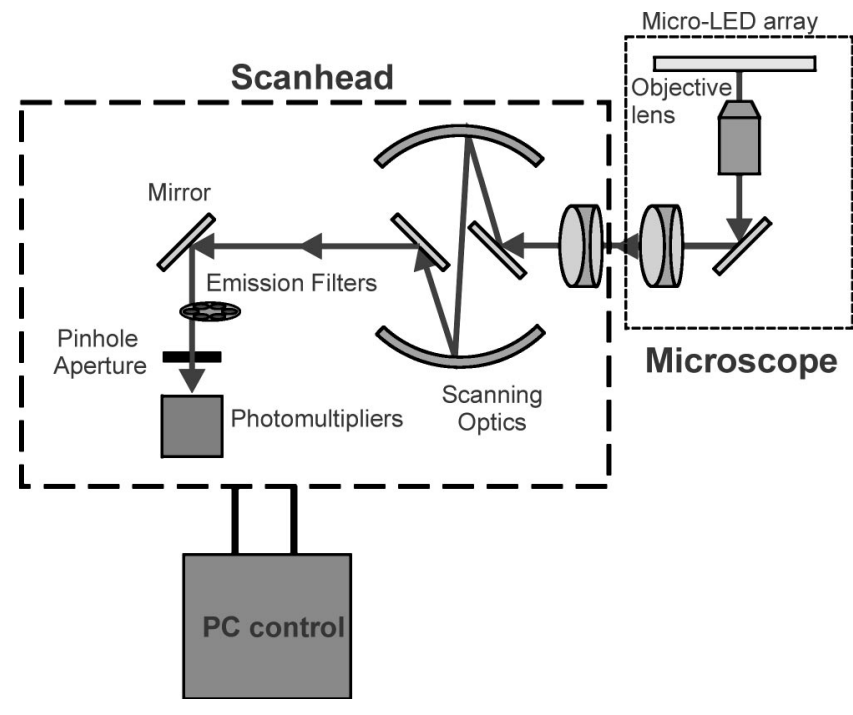

FIG. 1. Confocal microscope setup.

multipliers therein, the choice being determined by the emission wavelength of the device. The confocal aperture before the detector enabled optical sectioning of the LED output, as only light from the focal plane will reach the detector. Previous measurements ${ }^{9}$ had shown the axial and lateral resolutions of this system to be $0.8( \pm 0.1) \mu \mathrm{m}$ and $0.25( \pm 0.02) \mu \mathrm{m}$, respectively, at a wavelength of $488 \mathrm{~nm}$. In order to obtain the emission profiles, the system was scanned for the blue and green devices in sections in the $Z$ direction from -50 to $+50 \mu \mathrm{m}$, in steps of $1 \mu \mathrm{m}$, with the element surface designated as $0 \mu \mathrm{m}$. In the case of the UV device, the scan was taken from -25 to $+25 \mu \mathrm{m}$, due to the relatively low sensitivity of the photodetector to light below $400 \mathrm{~nm}$ wavelength. Care was taken to ensure that the detection system did not become saturated at any point during the scan. The images were then examined using commercial software. Each $X Y$ section was analyzed and an intensity histogram used to determine the full width at half maximum value for each plane. The values were then plotted in a standard plotting package and the beam divergence calculated from the resulting profile.

In a simple representation of the emission pattern with a 20- $\mu \mathrm{m}$-wide aperture, the LEDs may be expected ${ }^{10-12}$ to emit in a roughly Lambertian pattern, so to simulate the emission profile a Lambertian emission profile of the following form ${ }^{12}$ was used:

$$
I_{\text {air }}=\frac{P_{\text {source }}}{4 \pi r^{2}} \frac{n_{\text {air }}^{2}}{n_{s}^{2}} \cos \Phi .
$$

Here $I_{\text {air }}$ is the light intensity in air, $P_{\text {source }}$ is the total source power, $r$ is radial distance from the source, $n_{\text {air }}$ is the refractive index of air, $n_{s}$ is the semiconductor refractive in$\operatorname{dex}\left(n_{s}=2.456\right.$ for $\mathrm{GaN}$ at $\left.470 \mathrm{~nm}\right)$, and $\Phi$ is the angle of a ray refracted out of the surface. The emission distribution was simulated using 2000 source components in a $20-\mu \mathrm{m}$-wide line. Although not allowing for diffraction effects, the model provides a suitable system to assist in the interpretation of the recorded images under the conditions encountered here.

Reconstructed $X-Z$ sections through the center of the $X Y Z$ stacks of the measured device beam profiles are shown in Fig. 2. For clarity, negative intensity illustrations are used. (a)

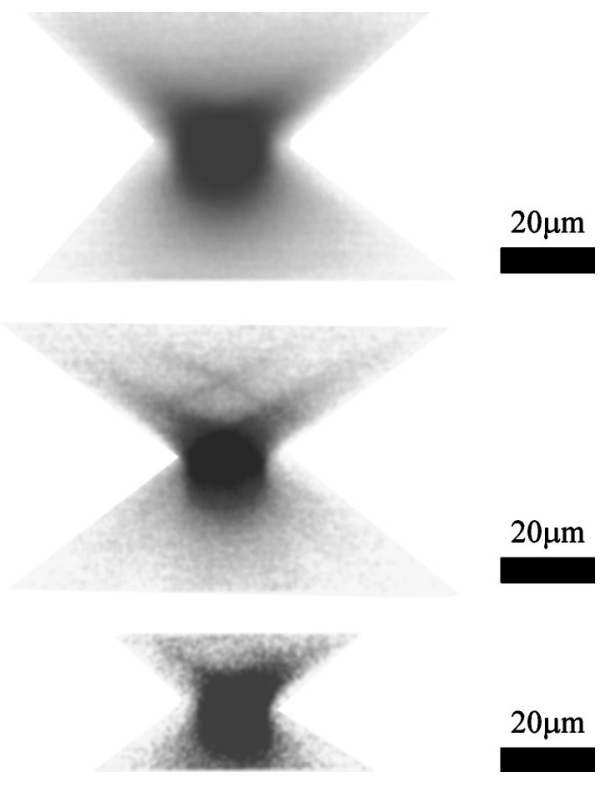

FIG. 2. Negative intensity $X-Z$ profiles of micro-LEDs, showing (a) blue device, (b) green device, and (c) UV device.

They show a clearly defined cone shape to the beams in the upward $(+Z)$ emitting direction, whilst light in the downward $(-Z)$ direction is still conical, but with a less well-defined distribution. This is likely to be due to photons emitting downwards from the quantum well scattering back up through the wafer.

As a representative example, intensity-mapped representations of the transverse intensity distribution from a blue device emission profile at $Z=10 \mu \mathrm{m}$ and $Z=30 \mu \mathrm{m}$, respectively, are shown in Figs. 3(a) and 3(b), along with the simulated results. Also shown are three-dimensional optical $X-Y$ sections. It should be noted that the intensity distributions are shown using spatial (microns) units rather than the angular distribution more normally used in LED emission profile measurements. ${ }^{10-12}$ This has been done to facilitate measurement of the second moment ${ }^{13,14}$ beam divergence values, and the results of Eq. (1) have been similarly expressed to allow a comparison between theory and measured values. All plots have been normalized with respect to the maximum mea-

(a)
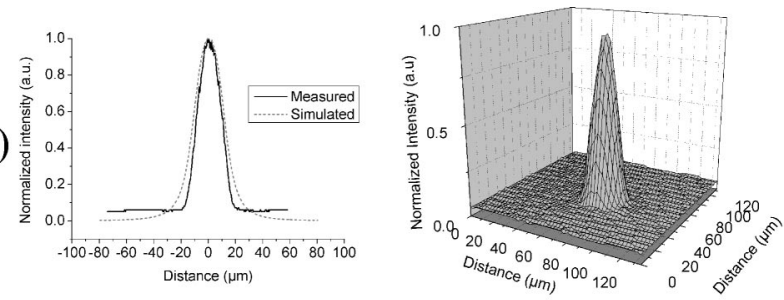

(b)
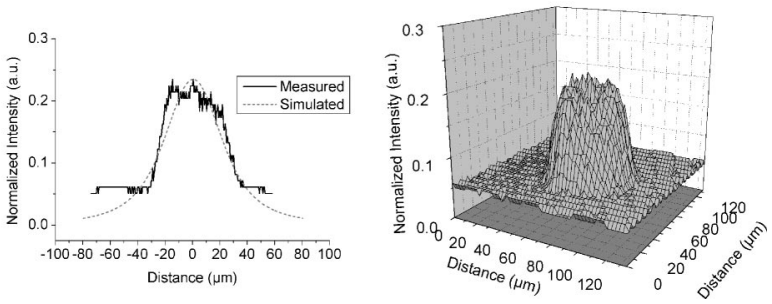

FIG. 3. Measured and simulated beam profiles of blue micro-LED at (a) $Z=10 \mu \mathrm{m}$ and (b) $Z=30 \mu \mathrm{m}$. Three-dimensional plots of the measured beam profiles at the same distances are shown on the right-hand side. 
TABLE I. LED beam profile results.

\begin{tabular}{cccc}
\hline \hline Color & Wavelength $(\mathrm{nm})$ & Objective n.a. & $\begin{array}{c}\text { Second moment } \\
\text { beam divergence } \\
\text { (full angle) }\end{array}$ \\
\hline Green & 510 & 0.75 & $72 \pm 5^{\circ}$ \\
Blue & 468 & 1.35 & $65 \pm 5^{\circ}$ \\
Blue & 468 & 0.75 & $70 \pm 5^{\circ}$ \\
UV & 370 & 0.75 & $75 \pm 3^{\circ}$ \\
Simulated & $\cdots$ & $\cdots$ & $71^{\circ}$ \\
\hline \hline
\end{tabular}

sured intensity value at $Z=10 \mu \mathrm{m}$, and, given the simplicity of the model, the simulated results provide a consistent fit to the measured intensity profiles. From the abrupt leveling-off of the measured line scans near zero, we can infer that most of the light emitted by the LED is contained within the cone shape seen in the $X-Z$ profiles.

The simulated plot at $Z=30 \mu \mathrm{m}$ is somewhat rounder than the measured plot, and noise in the measured plot contributes to its nonzero minimum. However, the simulated value at this distance is very similar to the measured value and thus gives a divergence result comparable to that of the actual LEDs. In addition, simulations of a $20-\mu$ m-wide disk of material with refractive index equal to $\mathrm{GaN}$ were performed using a commercially available nonsequential raytracing package. This produced a flat-topped emission profile at distances greater than $30 \mu \mathrm{m}$ from the disk when a reflective surface was positioned below the disk. This reflector has the effect of redirecting light emitted downward from the source back up through the disk and contributing to the overall emission profile, thereby forming the "top hat" profile similar to that seen in device measurements. Fresnel reflections from the sapphire substrate due to the difference in refractive index are likely to contribute to the observed beam shape in this way.

Table I shows second moment beam divergences measured for the three wavelengths of micro-LED array, together with the simulated result. It can be seen that the simulated results are in close agreement with the measured green and blue divergences. The small disparity with the UV device may be attributed to the smaller measurement range $( \pm 25 \mu \mathrm{m})$ of the UV profile. As a final verification that the confocal system was collecting all or most of the light from each element and therefore not giving a false divergence measurement, a 1.35 n.a. $\times 20$ oil-immersion lens, with oil of refractive index 1.51, was used to measure the divergence of a blue micro-LED element. The 1.35 n.a. lens is capable of collecting all the light within $128^{\circ}$ (full angle) emitted upwards by the micro-LED. The measurement using the 1.35 n.a. lens was actually smaller than divergence angles measured using the 0.75 n.a. lens, implying that the emitted light cone is not restricted by the 0.75 n.a. limiting full angle of $98^{\circ}$. Increasing the drive voltage on the blue device to $4 \mathrm{~V}$ did not significantly change its emission angle, implying a reasonable degree of independence of the observed profile to drive conditions.

In conclusion, we have measured the emission beam profiles of micrometer scale InGaN/GaN LEDs. Specific data have been obtained for devices emitting at blue, green, and ultraviolet wavelengths, respectively. Confocal microscopy has been utilized for this purpose, as a powerful means of resolving light emission from the devices. The technique provides a detailed image of the light distribution as opposed to conventional emission profile measurement techniques that are based entirely in the far field and therefore unsuitable for micro-optical elements. The knowledge gained should allow determination of the exact requirements for optimizing micro-optical elements in registry with the emitters, to manage the light emission for practical applications.

This work was supported by UK EPSRC and Scottish Enterprise. The authors would like to thank Nitride Semiconductor Co. Ltd. (Japan) for support with the UV wafers. Thanks go to Marijn Zandvliet of the Institute of Photonics for constructive discussions on second moment beam divergence measurement methods.

${ }^{1}$ H. X. Jiang, S. X. Jin, J. Li, J. Shakya, and J. Y. Lin, Appl. Phys. Lett. 78, 1303 (2001).

${ }^{2}$ I. Ozden, M. Diagne, A. V. Nurmikko, J. Han, and T. Takeuchi, Phys. Status Solidi B 188, 139 (2001).

${ }^{3}$ C. W. Jeon, H. W. Choi, and M. D. Dawson, IEEE Photonics Technol. Lett. 15, 1516 (2003).

${ }^{4}$ D. Graham, A. Grondin, C. McHugh, L. Fruk, and W. E. Smith, Tetrahedron Lett. 43, 4785 (2002).

${ }^{5}$ T. N. Oder, J. Shakya, J. Y. Lin, and H. X. Jiang, Appl. Phys. Lett. 82, 3692 (2003).

${ }^{6}$ S. H. Park, H. Jeon, Y. J. Sung, and G. Y. Yeom, Appl. Opt. 40, 3698 (2001).

${ }^{7}$ K. Okamoto, J. Choi, Y. Kawakami, M. Terazima, T. Mukai, and S. Fujita, Jpn. J. Appl. Phys., Part 1 43, 839 (2004).

${ }^{8}$ C. W. Jeon, H. W. Choi, and M. D. Dawson, Proceedings of the 16th Annual Meeting of IEEE LEOS, IEEE, Tucson, 2003, p. 878.

${ }^{9}$ J. M. Girkin, A. I. Ferguson, D. L. Wokosin, and A. M. Gurney, Opt. Express 7, 336 (2000).

${ }^{10}$ Z. Ogorelec and V. Radić, IEEE Trans. Electron Devices 38, 2062 (1991).

${ }^{11}$ S. J. Lee, Jpn. J. Appl. Phys., Part 1 37, 5990 (1998).

${ }^{12}$ E. F. Schubert, Light Emitting Diodes (Cambridge University Press, New York, 2003).

${ }^{13}$ K. C. Jorge, R. Riva, and N. A. S. Rodrigues, Annals of Optics, XXVI Brazilian National Meeting in Condensed Matter Physics (XXVI ENFMC), 2003, Vol. 5.

${ }^{14}$ International Standards Organization for Standardization, Test Methods for Laser Beam Parameters: beam widths, divergence angle and beam propagation factor, in ISO 11146, 1999. 